\title{
Diagnosis of Ball Bearing Faults Using Double Decomposition Technique
}

\author{
T. Dovedi \\ Thapar Institute of Engineering and Technology, Patiala, Punjab, India. \\ R. Upadhyay \\ Thapar Institute of Engineering and Technology, Patiala, Punjab, India. \\ Trinity College Dublin, Ireland.
}

\begin{abstract}
(Received 19 March 2019; accepted 2 August 2019)
The rolling element bearing is one of the most significant components of any rotating machinery. However, the foremost cause of malfunction in any rotating machine is due to defects like cracks, dents, spall, pits, etc. in ball bearings. Early diagnosis of these bearing faults is highly essential to avoid an accidental shutdown of rotating machinery. In the present work, a novel technique of bearing fault diagnosis is proposed following double decomposition of the vibration activity. The experimentally recorded vibration signals are processed through two stages of decomposition viz. Empirical Mode Decomposition and Tunable Q-factor Wavelet Transform based Time-Frequency decomposition. Subsequently, sub-bands of decomposed time-frequency activity are acquired and discriminable features are computed. Fractal Dimension (FD) based features are extracted from each decomposed sub-band as complexity measures of time-frequency sub-bands. In order to classify bearing faults, a Support Vector Machine classifier is trained with acquired features and classification performance is evaluated. The results of classification reveal that the proposed double decomposition technique is a potential candidate in extracting viable vibration signatures for fault identification. The study is conducted on Case Western Reserve University bearing datasets.
\end{abstract}

\section{INTRODUCTION}

The rolling element bearing is a component of great significance used in manufacturing industry. These bearings undergo unexpected failures by the impacts of corrosion, wear, fatigue and overload conditions. Unexpected failure in a bearing results in sudden shut down of machinery and in turn causes heavy loss to the manufacturing industry. This is the reason why autonomous bearing fault diagnosis has gained major attention of researchers across the globe. Efficient bearing fault diagnosis using vibration signal analysis can prevent sudden failure of the bearings and cut down the cost of repair and shut down of machinery. However, vibration signal based bearing fault detection is a challenging task, since vibration signals are non-stationary and non-linear in nature and are often severely affected by the external noise from other parts of the machines. This makes it obligatory to exploit an efficient fault diagnosis methodology in order to generate highly discriminative features. Feature extraction and classification of given vibration activity are the two vital steps in the bearing fault diagnosis task. $^{1}$

In the past, various signal processing techniques were proposed for diagnosing faults in the bearings. The earliest signal processing techniques of fault diagnosis were simple and were mainly dependent on calculation of statistical parameters viz. mean, kurtosis, root mean square features from the time series data. For instance, the use of normalized kurtosis and normalized skewness values as features of vibration signals was suggested by Honarvar and Martin for early fault diagnosis. ${ }^{2}$ Honarvar and Martin also used statistical moments in rolling element bearing health monitoring. ${ }^{3}$ In another work, Mechefske and Mathew proposed the use of an autoregressive model for bearing fault diagnosis purposes. ${ }^{4} \mathrm{Li}$ and $\mathrm{Qu}$ developed a signal demodulation technique using the concept of cyclic spectrum and cyclic autocorrelation for recovering fault information present in the modulators of bearing vibration signals. ${ }^{5}$ Considering the multi-dimensional and multiscale nature of vibration signals, Zvokelj developed an approach by combining the Ensemble Empirical Mode Decomposition (EEMD) method with Principal Component Analysis (PCA) to analyze the non-stationary behavior of bearing signals. ${ }^{6}$ However, these methods were not capable of minimizing the effect of interference and noise generated from other parts of the machines. In addition, these methods failed to provide any additional information about the frequency content of the signals.

In order to capture information content from both time and frequency domains, researchers suggested use of timefrequency transformation techniques viz. Wigner-Vile distribution, Short Time Fourier Transform (STFT), Hilbert-Huang Transform (HHT) and Wavelet Transform (WT) for bearing fault diagnosis. For instance, Mori et al. introduced a Discrete Wavelet Transform (DWT) based feature extraction technique to predict spalling of ball bearings. ${ }^{7}$ In another work, Seker and Ayaz suggested WT based Multi-Resolution Analysis (MRA) for diagnosing faults of electric motors. ${ }^{8}$ Lou and Loparo used WT based feature vectors drawn from normalized vibration signals to train an adaptive neural-fuzzy inference system for classification purposes. ${ }^{9}$ In similar work, Yang et al. applied an HHT to decompose vibration activity of faulty bearings and extracted substantial features. ${ }^{10}$ In another work, He et al. proposed a hybrid method of Morlet wavelet filtering and sparse code shrinkage for the detection of impulses generated from bearing faults. ${ }^{11}$ Researchers also applied several other supplementary methods viz. histograms, complexity measures and entropy based schemes to retrieve fault information from 
vibration activity spawned by bearings. For instance, Wyk et al. used Difference Histograms for extracting faulty features of rolling element bearing vibration activity. ${ }^{12}$ In another work, Hong and Liang employed normalized Lempel-Ziv complexity values to assess the fault severity of a rolling element bearing. ${ }^{13}$

In recent years, various modern feature extraction techniques have been proposed by the researchers. For instance, Zhao et al. employed an Empirical Mode Decomposition (EMD) technique with Approximate Entropy (AE) features for this purpose. ${ }^{14}$ In similar work, Dybala and Zimroz Rolling developed a bearing diagnosing methodology based on EMD of machine vibration signals. ${ }^{15}$ In another work, Liu et al. introduced a novel methodology of feature extraction, stated as a Local Mean Decomposition (LMD) technique, in which vibration activity was decomposed into a series of product functions defined as the product of an amplitude envelope signal and a subsequent frequency modulated signal. ${ }^{16}$ The method of Hilbert Transformation (HT) supplemented by the Duffing oscillator for recognizing localized defects in ball bearings was suggested by Patel et al. ${ }^{17}$ A new dimension in this area was added by Klein et al. by applying image processing techniques viz. ridge tracking on the Time-Frequency Representation (TFR) of the bearing vibration signals. ${ }^{18}$ In another work, Ming et al. introduced a new technique of Spectral Auto-Correlation Analysis (SACA) by performing autocorrelation on the Fast Fourier Transform (FFT) transformed signals for fault diagnosis of rolling element bearings. ${ }^{19}$ Lei et al. suggested an improved kurtogram methodology in combination with Daubechies-Wavelet based Wavelet Packet Transform (WPT) filtering for this purpose. ${ }^{20}$ In another work, Li et al. suggested the Continuous Wavelet Transform (CWT) based reassigned Wavelet Scalogram (WS) technique of bearing fault diagnosis. $^{21}$

With the advancement of nonlinear dynamic modelling, studies advocating nonlinear parameter estimation from bearing vibration signals have been suggested in the recent past and been proven very effective in bearing fault diagnosis. Such studies extract non-linear information hidden in the vibration activity by estimating a range of nonlinear dynamic parameters viz. correlation dimension, ${ }^{22}$ Approximate Entropy (ApEn), ${ }^{23}$ Permutation Entropy (PE) ${ }^{24}$ and Multiscale Entropy (MSE). ${ }^{25}$ The advancement in signal processing techniques impelled bearing fault diagnosis research widely. Various significant and effective feature estimation methods have been developed in recent years. For instance, Liang and Faghidi proposed an intelligent fault identification method using a calculus enhanced energy operator. ${ }^{26}$ Caesarendra et al. developed a largest Lyapunov exponent algorithm to estimate the degree of non-linearity for low speed slew bearing condition monitoring. ${ }^{27}$ In order to anticipate the Remaining Useful Life (RUL) of bearings, Boskoski et al. proposed bearing fault prognostics method using Renyi's entropy and Gaussian process model. ${ }^{28}$ Considering non-linearity, non-stationarity and multifractal properties of vibration signals, a method based on Local Characteristic-scale Decomposition-Teager Energy Operator (LCD-TEO) and Multifractal Detrended Fluctuation Analysis (MF-DFA) was first proposed by Liu et al. ${ }^{29}$ In another work, Han et al. ${ }^{30}$ suggested a feature extraction method using improved Fast-ICA (Independent Component Analysis) algorithm and the wavelet packet energy spectrum. In order to identify multi-faults in rotating machinery a method combining Spectral Kurtosis (SK) and Minimum Entropy Deconvolu- tion (MED) techniques was proposed by He et al. ${ }^{31}$ A kurtosis based weighted sparse model with a convex optimization technique and Multipoint optimal MED based bearing fault diagnosis techniques are proposed in more recent work. ${ }^{32,33}$ Other entropy-based bearing fault diagnosis methods include composite multiscale fuzzy entropy ${ }^{34}$ and fusion information entropy-based feature extraction. ${ }^{35}$ In similar work, an improved maximum correlated kurtosis deconvolution method was suggested by Miao et al. ${ }^{36}$

Due to its excellent band pass filtering characteristics, the WT has been extensively used in many fault diagnosis applications in the past. However, WT suffers from the problem of constant Q-factor i.e. it has a constant central frequency to bandwidth ratio. In order to overcome shortcomings of classical WT, Selesnick proposed the concept of Tunable Q-factor Wavelet Transform (TQWT). ${ }^{37}$ In another work, Cai et.al suggested the concept of Sparsity-enabled signal decomposition using TQWT for fault feature extraction of a gearbox. ${ }^{38} \mathrm{~A}$ TQWT has also been used in combination with a Hilbert transform for investigating the vibration features of motor bearing faults. ${ }^{39}$ Weak bursts of vibration signals of an angular contact bearing were extracted by Kumar et.al using TQWT along with envelope demodulation. ${ }^{40}$ In the present work, the capabilities of TQWT are further extended using EMD in addition to TQWT. The proposed double decomposition technique ensures frequency bound TQWT coefficient estimation and helps in detailed analysis of decomposed high frequency vibration activity. An EMD technique decomposes given vibration activity into set of signals of specific frequency known as Intrinsic Mode Functions (IMFs). Thereafter, TQWT is applied onto specific set of IMFs with high Fractal Dimension (FD) value and TQWT coefficient sub-bands are attained. Further, Higuchi's Fractal Dimension (HFD) estimation is carried out on decomposed sub-bands for extraction of useful vibration signatures and preparation of the feature vector. The obtained feature vector is fed as input to a soft computing technique for training and validation purposes. In the present work, a Support Vector Machine (SVM) classifier is employed for fault classification purposes. The results of the classification revealed that proposed double decomposition-based fault classification methodology gives better results than the conventional methods used for bearing fault diagnosis in the past. The organization of the present work is as follows: Section 2 provides a detailed description of the material and methods being used in carrying out the present study. The description of proposed double decomposition-based feature extraction methodology is presented in section 3 . The outcomes of the study are discussed in section 4 following by conclusion in section 5 .

\section{METHODS AND MATERIAL}

\subsection{Experimental Dataset}

The bearing vibration data used and analyzed in the present work is obtained from the Case Western Reserve University bearing data center. Bearing faults ranging between 0.007 inches to 0.040 inches $(1$ inch $=25.4 \mathrm{~mm}$ ) in diameter were introduced in the rolling element, inner raceway and the outer raceway using electro-discharge. All the data files being used are in MATLAB format. Each file contains drive end and fan end vibration data along with the motor rotational speed. Four classes of data are considered in this study namely, Healthy Bearing (HB), Ball Defect (BD), Inner Race defect (IRD) and Outer Race Defect (ORD). However, the data of HB 


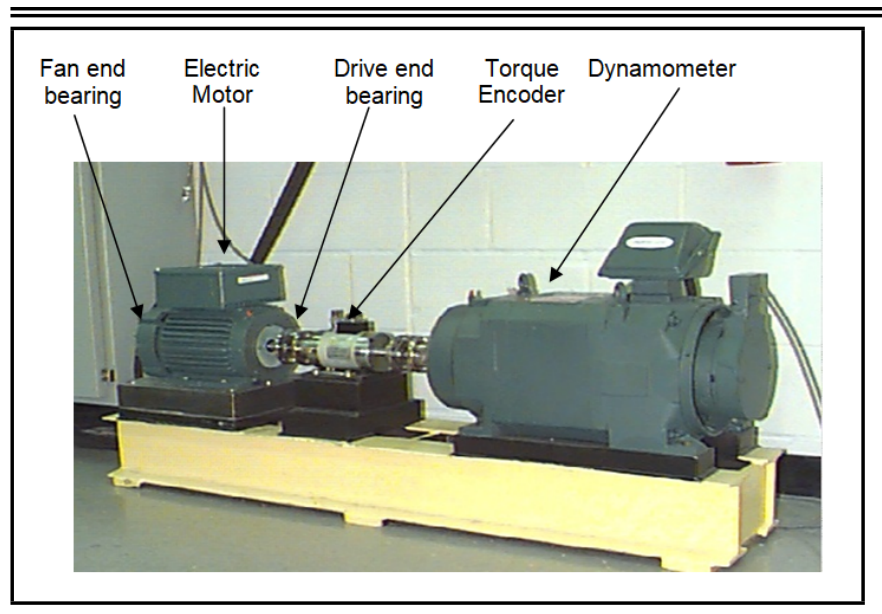

Figure 1. Experimental Test Rig for ball-bearing vibration analysis

is considered as the baseline data. Bearing data was collected at sampling rates of $48 \mathrm{kHz}$ and $12 \mathrm{kHz}$ from drive end and fan end. The data is registered at four different speeds of ball bearing i.e. 1730, 1750, 1772 and $1797 \mathrm{rpm}$. The faults considered in present study includes IRD, ORD at 6 o'clock position and BD with defect sizes of 7 mils, 14 mils and 21 mils $(1 \mathrm{mil}=$ 0.001 inch). Figure 1 shows the experimental test rig used in acquiring bearing vibration data. A sample plot of four classes of bearing vibration signals are represented in Fig. 2.

\subsection{Empirical Mode Decomposition (EMD)}

EMD was proposed by Huang et al. in 1998. ${ }^{41}$ EMD was developed on the assumption that any time series consists of different simple intrinsic modes of oscillations. It is a self adaptive signal decomposition technique which decomposes any time series into different oscillation modes and the original signal $s(t)$ can be recovered by a linear superposition of empirical modes as:

$$
s(t)=\sum_{i=0}^{n} e_{i}(t)+r_{n}(t)
$$

where $e_{i}(t)$ is the $i^{t h}$ empirical mode and $r_{n}(t)$ is the final residue after estimation of $n$ empirical modes. The EMD technique is very useful, particularly in the analysis of nonlinear and non-stationary vibration activity, since it decomposes the original vibration signal into simple oscillatory functions called IMFs $e_{i}(t)$, while following set of conditions. ${ }^{41}$

1. The number of extrema and the number of zero crossings must either be equal or differ by at most one.

2. The mean value of the envelopes defined by local maxima and minima at any point should be zero.

Given two conditions ensure that for any function to be an IMF all its local maxima should be positive and local minima should be negative. ${ }^{42}$ The decomposition of any input signal $s(t)$ into IMFs is attained by a shifting process, which can be summarized as:

1. Identify all extrema (i.e. maxima and minima) of the given input signal $s(t)$.

2. Connect extrema (i.e. maxima and minima) separately with cubic spline interpolation and generate upper $\left(e_{\max }(t)\right)$ and lower envelopes $\left(e_{\min }(t)\right)$ to cover complete data between envelopes.
3. Estimate the running mean $m_{1}(t)$ between upper and lower envelopes. The difference between $m_{1}(t)$ and the signal $s(t)$ is the component $I_{1}(t)$. If $I_{1}(t)$ satisfies condition of IMF, then it is the first component of $s(t)$.

4. If $I_{1}(t)$ doesn't satisfies the conditions, consider $I_{1}(t)$ as the original signal $s(t)$ and repeat steps 1-3 until first IMF is obtained. After repeated sifting for $j$ times, first IMF $I_{1 j}(t)$ (represented as $c_{1}(t)$ ) is obtained as:

$$
I_{1(j)}=I_{1(j-1)}-m_{1 j}=c_{1}(t) .
$$

The obtained IMF $c_{1}(t)$ represents smallest temporal component of the signal $s(t)$. Further, in order to attain other IMFs, residue $r_{1}(t)$ is generated by subtracting $c_{1}(t)$ from signal $s(t)$. Here, $r_{1}(t)$ is treated as the original data and the process is repeated to attain second IMF component $c_{2}(t)$. The decomposition process continues until the final residue is a constant, a monotonic function or a function from which no other IMFs can be derived. All derived IMFs (i.e., $c_{1}(t), c_{2}(t), \ldots, c_{n}(t)$ ) represent specific frequency band ranging from high to low and are stationary in nature. Once decomposition process completes, original signal $s(t)$ can be obtained as:

$$
s(t)=\sum_{n=1}^{n} c_{n}(t)+r_{n}(t)
$$

where $n$ signifies number of IMFs, $c_{n}(t)$ is the $n^{t h}$ IMF, and $r_{n}(t)$ represents the residue of the decomposition process. Figure 3 and Fig. 4 represent the IMFs obtained after decomposition of healthy and ball defect vibration activity consecutively. It is evident from Fig. 3 and Fig. 4 that higher order IMFs effectively epitomize lower frequencies and lower order IMFs are suitable for higher frequencies.

Despite the fact that vibration activity of faulty bearing comprises high information content as compared to healthy bearing, a lower number of IMFs (i.e. 16 IMFs) are required in the decomposition process. Theoretically, a larger number of IMFs should be generated after decomposition of faulty bearing vibration activity, however, this not the case always, as notified by Dybala and Zimroz. ${ }^{43}$ Vibration activity suffers environmental and instrumental noise which largely reflect in some IMFs and the number of decomposed IMFs significantly depend on the amount of noise present in any vibration activity. Since, early stage fault signatures are well submerged into the system noise, it makes early stage fault diagnosis a challenging task. In recent years, researchers proposed various EMD based hybrid fault diagnosis techniques and reported substantial diagnostic results. ${ }^{44}$ Hybrid EMD techniques in addition to artificial intelligence have shown promising results in the previous studies. ${ }^{45}$ In the present work, the EMD algorithm proposed by Rato et al. is implemented to estimate IMFs of vibration signals. ${ }^{46}$ Rato et al. suggested that reducing the resolution factor reduces the number of obtained IMFs. This property is useful for sidewise analysis of different signals decomposed into the same number of IMFs. In order to optimize resolution factor, it is varied in the band of $40-50 \mathrm{~dB}$ with step size of $5 \mathrm{~dB}$ and experimental results are evaluated for attaining the highest classification performance. The proposed automatic bearing fault diagnosis methodology using EMD based double-decomposition reduces noise influences on the decision system's performance by treating decomposed IMFs with TQWT. 


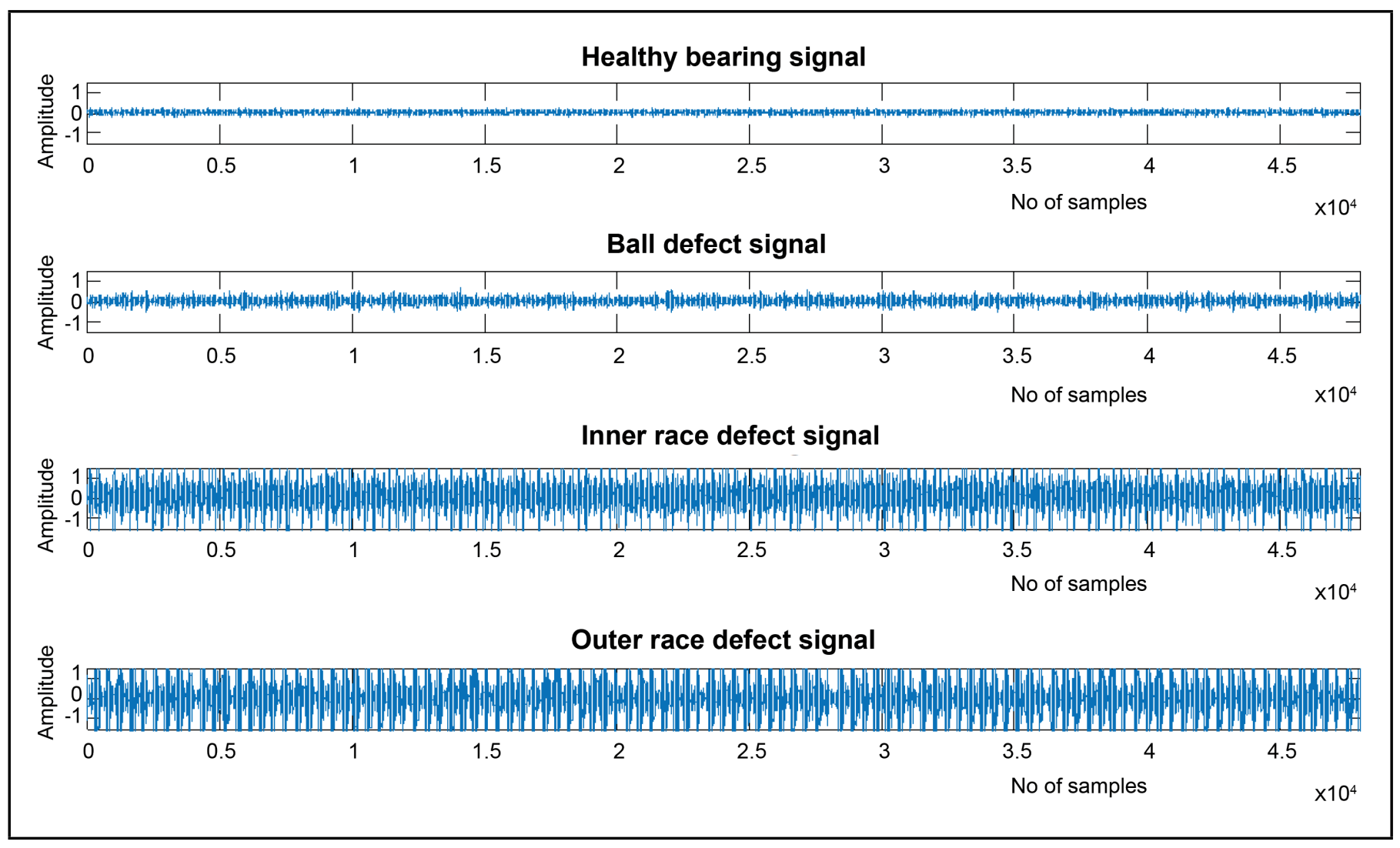

Figure 2. Sample plot of four classes of bearing vibration data.

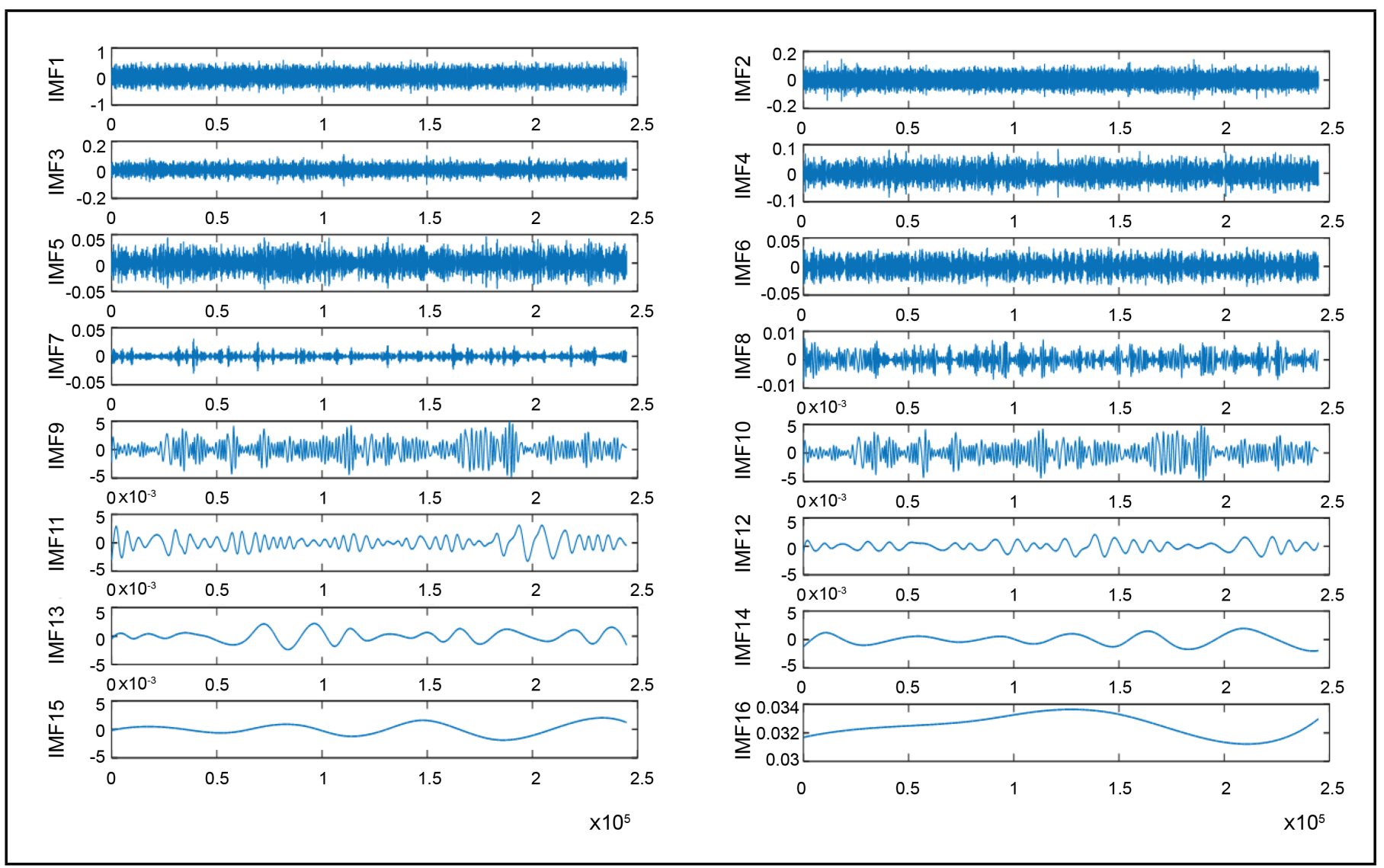

Figure 3. IMFs obained after applying EMD on ball defect vibration signal. 

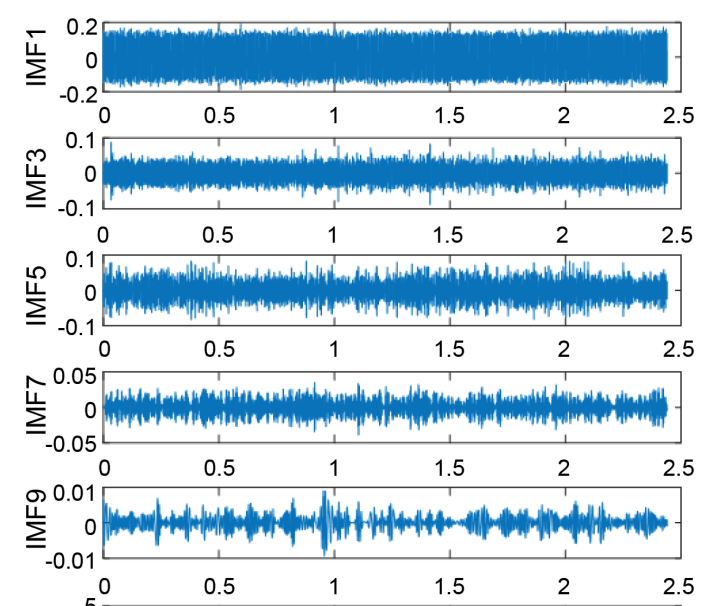

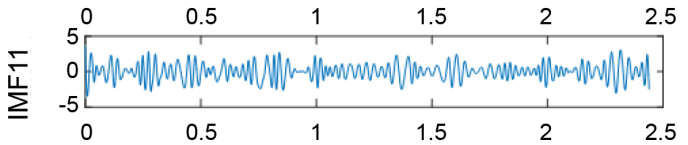
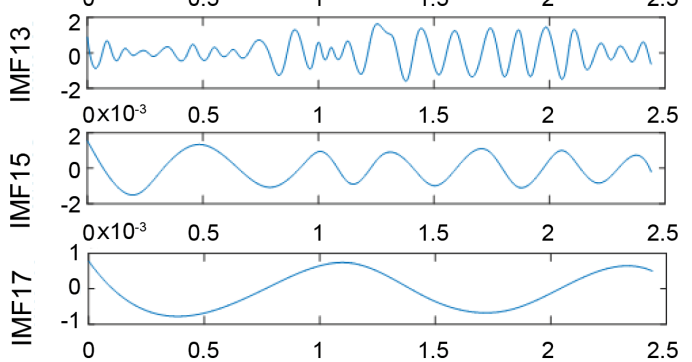
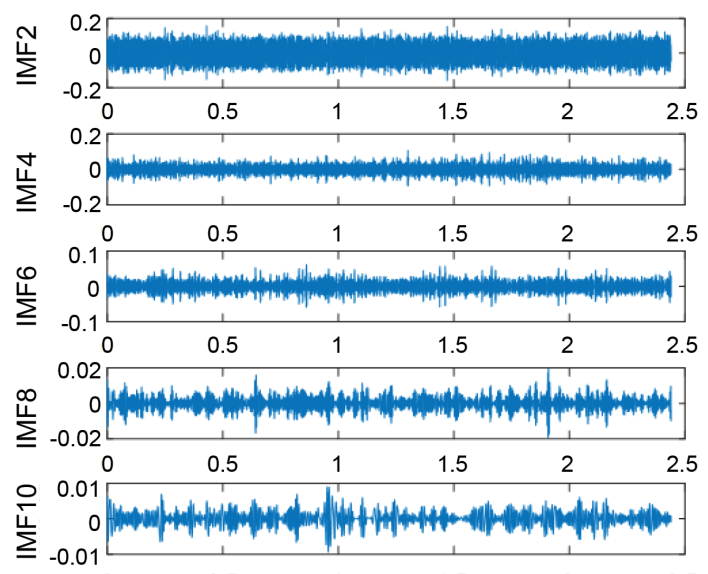

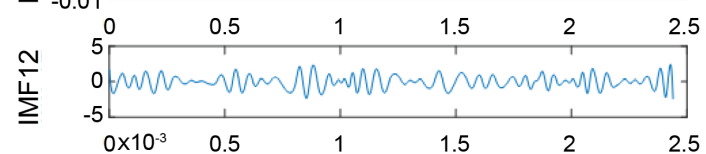
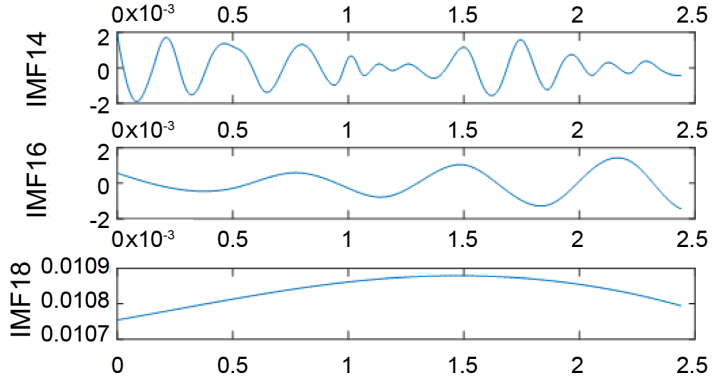

Figure 4. IMFs obained after applying EMD on healthy vibration signals.

\subsection{Tunable Q-factor Wavelet Transform (TQWT)}

The DWT is a commonly used TFR technique for bearing fault diagnosis, since, it is capable of apprehending disparities in the morphologies of bearing vibrational activity. ${ }^{47}$ The TQWT is the expansion of the DWT in terms of parametric values adjustment ability to attain the desired Time-Frequency response. The parameters of TQWT includes Quality-factor (Qfactor $(Q)$, redundancy parameter $(r)$ and decomposition levels $(p)$. The parameter $Q$ controls the number of oscillations, $r$ limits unwanted excessive ringing and ensures localization of the wavelet in the time domain with preserved shape characteristics. ${ }^{37}$ The filters of TQWT have a non-rational transfer function and maintain a direct relationship with the frequencydomain. This property of TQWT helps in gaining perfect reconstruction ability and proficient implementation using an FFT algorithm. ${ }^{48}$ The decomposition of vibration activity into specific number of levels (i.e. $p$ levels) is achieved by iteratively smearing two channel filter banks onto given vibration activity. As shown in Fig. 5, the input signal $s(n)$ (having sampling frequency $\left.=f_{s}\right)$ is decomposed into low-pass $\left(l^{0}(n)\right)$ and high-pass $\left(h^{1}(n)\right)$ sub-band signals with altered sampling frequencies of $\alpha f_{s}$ and $\beta f_{s}$, respectively. The low-pass signal $l^{0}(n)$ is generated by passing $s(n)$ through low pass filter $H_{0}(\omega)$ followed by low-pass scaling factor, LPS $\alpha$. Similarly, high-pass signal $h^{1}(n)$ is generated by passing $s(n)$ through a the high-pass filter $H_{1}(\omega)$ followed by scaling factor, HPS $\beta$. It is established in the literature that, the value of scaling parameters should follow the condition given as: $0<\beta \leq 1$; $0<\alpha<1 ; \alpha+\beta=1$; to ensure controlled redundancy and perfect reconstruction of signals.

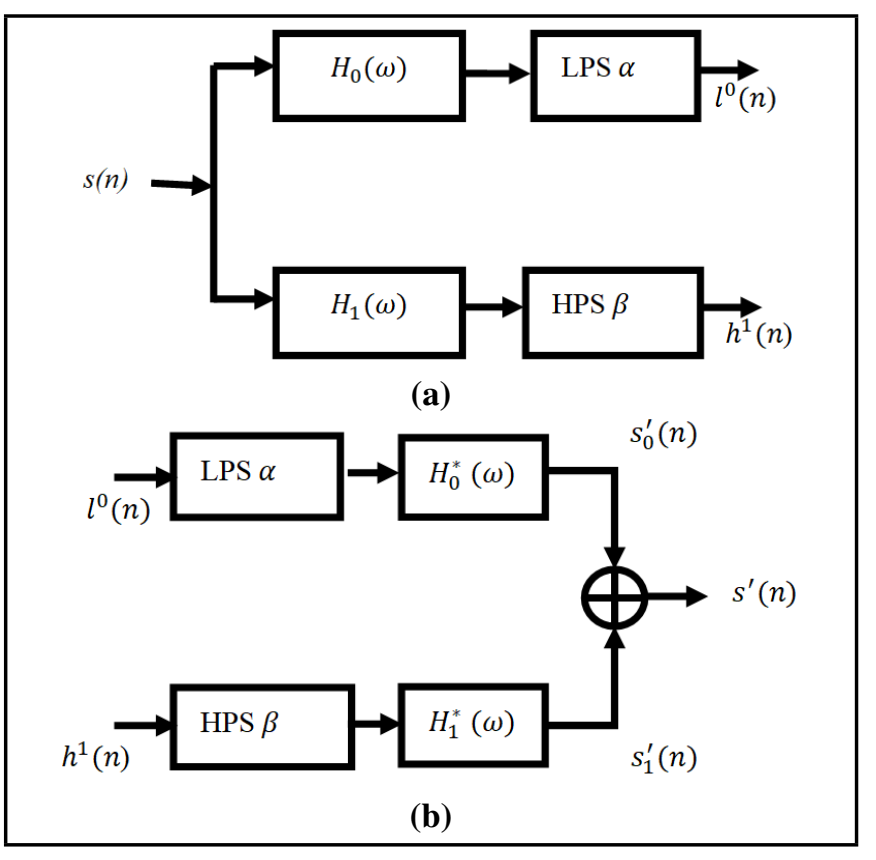

Figure 5. Decomposition of vibration activity in single level (a) Analysis filter bank (b) Synthesis filter bank.

In TQWT, for a given value of Q-factor, with an increase in the number of decomposition levels $(j)$, the cut-off frequency and bandwidth reduce. However, with the increment in Qfactor value, the frequency response compresses and it requires more decomposition levels to cover the entire frequency range as depicted in Fig. 6. In order to attain optimum TFR, the value of Q-factor should be maintained low for little or no oscillatory vibrational activity. On the other side, a high Q-factor 


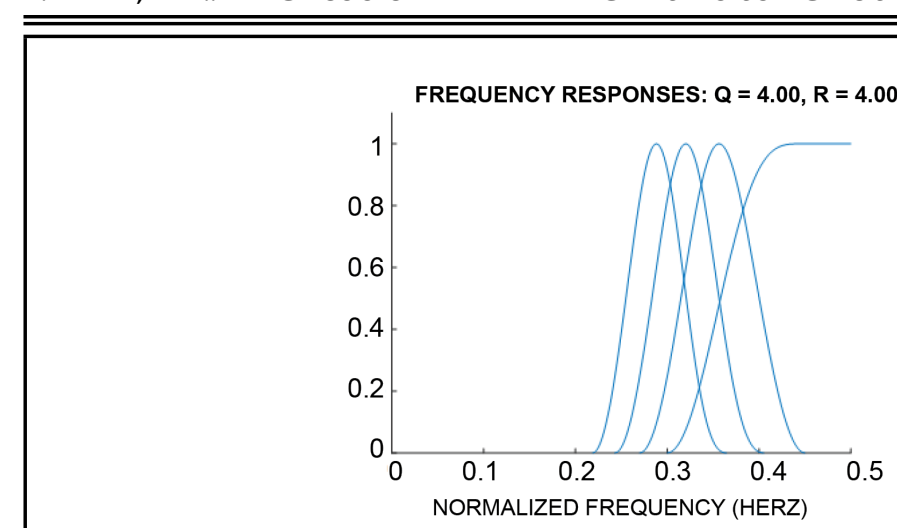

(a)

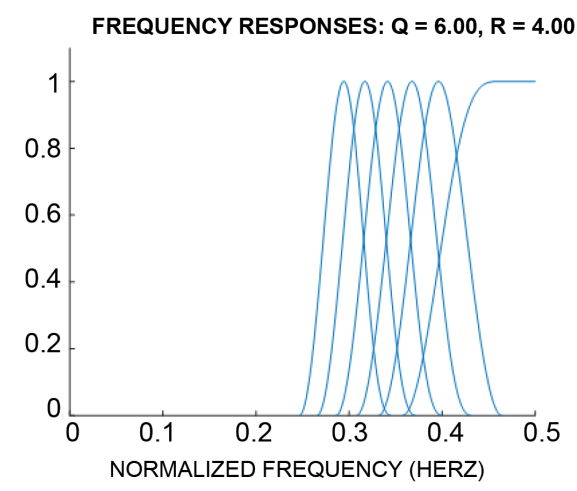

(C)

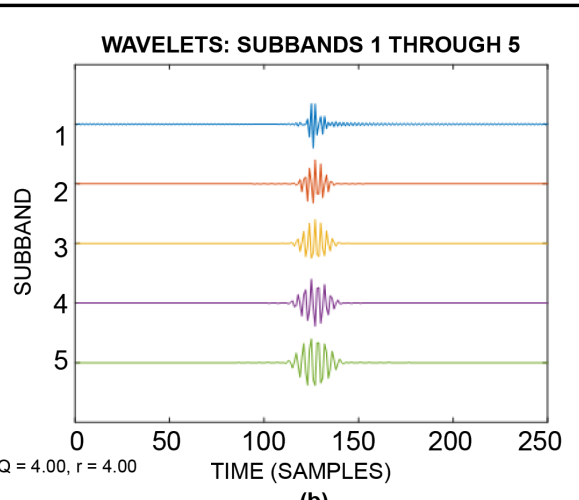

(b)

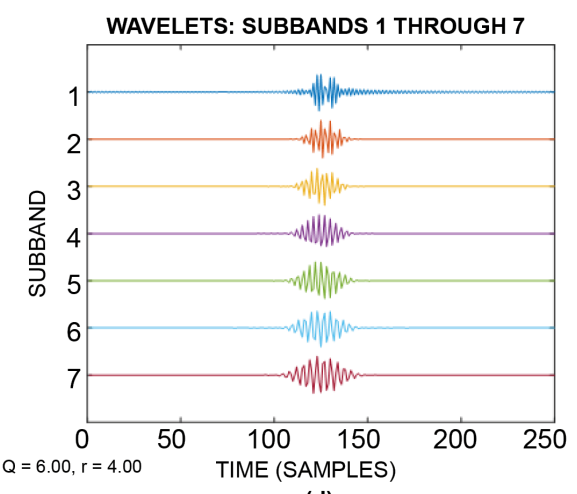

(d)

Figure 6. Effect of Q-factor value on (a-b) the normalized frequency response of TQWT and (c-d) corresponding sub-bands; for (a) $Q=4, j=4$ and $r=4$ (b) $Q=6, j=6$ and $r=4$.

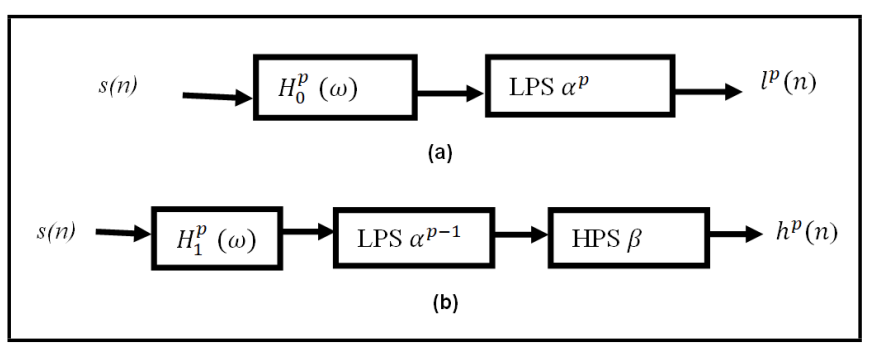

Figure 7. The identical system to yield coefficients of (a) low-pass sub-band $l^{p}(n)$ (b) high-pass sub-band $h^{p}(n)$.

value is required to analyze high oscillatory vibrational activities. However, other than CWT, WTs have less capability to adjust its Q-factor value. ${ }^{37}$ The ability of parameter adjustment offered by TQWT makes it an expedient tool for nonstationary, oscillatory vibration signal analysis.

The identical system to yield coefficients of low-pass and high-pass sub-bands (i.e. $l^{p}(n)$ and $h^{p}(n)$ ) with $p^{t h}$ number of decomposition levels is presented in Fig.. 7. The identical frequency responses of low-pass and high-pass sub-band filters (i.e. $H_{1}^{p}(\omega)$ and $H_{0}^{p}(\omega)$ ) with $p^{t h}$ level of decomposition is presented in Fig. 7(a), and is expressed as:

$$
\begin{gathered}
\begin{cases}\prod_{\dot{m}=0}^{p-1} H_{0}\left(\frac{\omega}{\alpha^{m}}\right) & |\omega| \leq \alpha^{p} \pi \\
0 & \alpha^{p} \pi<|\omega| \leq \pi\end{cases} \\
\begin{cases}H_{1}\left(\frac{\omega}{\alpha^{p-1}}\right) \prod_{\dot{m}=0}^{p-2} H_{0}\left(\frac{\omega}{\alpha^{\dot{m}}}\right) & (1-\beta) \\
0 & \alpha^{p-1} \pi \leq|\omega| \leq \alpha^{p-1} \pi .\end{cases} \\
\text { for other } \omega \in[-\pi, \pi]
\end{gathered}
$$

Figure 5(b) shows synthesis filters engaged in the reconstruction of decomposed vibration activity. The relationship be- tween scaling parameters (i.e. $\alpha$ and $\beta$ ) and TQWT input parameters is expressed by Eq. (6) and Eq. (7) as:

$$
\begin{aligned}
& r=\frac{\beta}{1-\alpha} ; \\
& Q=\frac{2-\beta}{\beta} .
\end{aligned}
$$

In TQWT based techniques, identification of suitable number of decomposition levels is a crucial part. However, in present study, criterion of dominant frequency is used to attain suitable number of decomposition level. Number of decomposition levels are selected in a way that the decomposed subband frequencies correlated well with the frequency ranges of interest (i.e. ball spin frequency/or ball pass frequency). It is perceived in this study that higher number of decomposition levels help in extracting highly differentiable fractal features from IMFs with variable frequency ranges. Decomposing IMFs into higher number of levels (i.e. $j=9$ ) gives better insight into the frequency ranges of interest. In the present work, the redundancy parameter $r$ is selected as $r=3$. Keeping the redundancy parameter $r \geq 3$ helps in correct localization of the Wavelet's response in time-domain and broadening the transition bands of low pass and high pass sub-band filters. Hongchao et al. notified that classical Wavelet Transform (WT) with constant Q-factor is inefficient in handling vibrational activity having significantly high/low Q-factor values. ${ }^{34}$ Therefore, in order to obtain appropriate value of Qfactor, multiple experiments are carried out on vibration data with varying Q-factor in the range of 1-50 in this work. 


$$
L(m, k)=\frac{\left\{\left(\sum_{i-1}^{i n t((N-m) / k)}|x[m=i k]-x[m+(i-1) \times k]|\right)\left[(N-1) /\left(\operatorname{int}\left(\frac{N-m}{k}\right) \times k\right)\right]\right\}}{k} .
$$

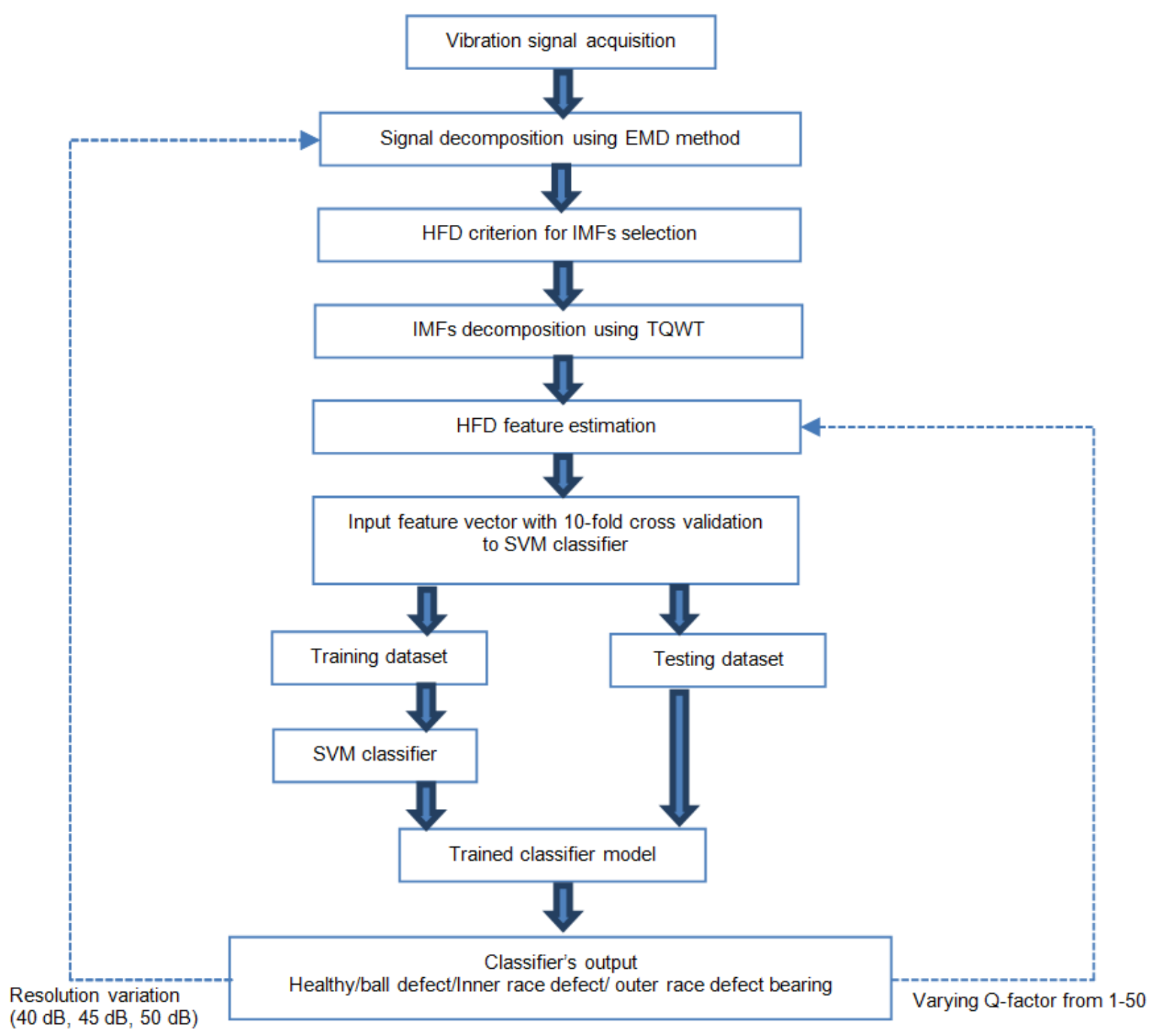

Figure 8. Schematic diagram of proposed methodology of fault diagnosis.

Table 1. Sample input feature vector obtained from processing of single IMF.

\begin{tabular}{|c|c|c|c|c|c|c|c|c|c|c||}
\hline F1 & F2 & F3 & F4 & F5 & F6 & F7 & F8 & F9 & F10 & CLASS \\
\hline 1.76 & 1.65 & 1.66 & 1.81 & 1.47 & 2.02 & 1.88 & 1.63 & 1.75 & 1.40 & HB \\
\hline 1.76 & 1.66 & 1.69 & 1.75 & 1.47 & 2.00 & 1.88 & 1.65 & 1.74 & 2.00 & HB \\
\hline 1.71 & 1.66 & 1.69 & 1.77 & 1.47 & 1.99 & 1.88 & 1.63 & 1.75 & 2.03 & HB \\
\hline 1.72 & 1.69 & 1.70 & 1.89 & 1.72 & 1.59 & 1.79 & 1.78 & 1.69 & 1.11 & BD \\
\hline 1.73 & 1.69 & 1.74 & 1.85 & 1.71 & 1.59 & 1.78 & 1.81 & 1.68 & 1.12 & BD \\
\hline 1.74 & 1.69 & 1.73 & 1.85 & 1.72 & 1.58 & 1.78 & 1.83 & 1.68 & 1.13 & BD \\
\hline 1.71 & 1.68 & 1.73 & 1.73 & 1.75 & 1.61 & 1.86 & 1.83 & 1.70 & 1.21 & IRD \\
\hline 1.75 & 1.70 & 1.73 & 1.70 & 1.76 & 1.57 & 1.87 & 1.84 & 1.66 & 1.29 & IRD \\
\hline 1.79 & 1.71 & 1.75 & 1.71 & 1.74 & 1.60 & 1.85 & 1.88 & 1.61 & 1.36 & IRD \\
\hline 1.79 & 1.70 & 1.66 & 1.85 & 1.69 & 1.53 & 1.87 & 1.65 & 1.65 & 1.12 & ORD \\
\hline 1.72 & 1.72 & 1.67 & 1.86 & 1.67 & 1.55 & 1.87 & 1.64 & 1.63 & 1.11 & ORD \\
\hline 1.76 & 1.73 & 1.66 & 1.86 & 1.65 & 1.55 & 1.89 & 1.62 & 1.67 & 1.11 & ORD \\
\hline
\end{tabular}

HB: Healthy Bearing, BD: Ball Defect, IRD: Inner Race Defect, ORD: Outer Race Defect ,F1:HFD of 1st sub-band of selected IMF, F2: HFD of 2nd sub-band of selected IMF 


\section{PROPOSED METHODOLOGY OF FRACTAL FEATURE ESTIMATION}

In the present work, each vibration signal is treated individually and corresponding features are estimated. On arrival of each vibration signal to the input of the algorithm, its HFD is calculated as the feature of the signal after performing EMDTQWT based double decomposition. Once, desired HFD features are estimated from all vibration signals available in the bearing dataset, the feature vectors with varying sizes are prepared and classification is performed. The proposed methodology of double decomposition is carried out in three methodological steps. In the first step, segments of raw vibration signals are decomposed into IMFs of specific frequency using an EMD technique. Thereafter, TQWT is applied on the selected IMFs to attain time-frequency coefficients of the decomposed IMFs. Subsequently, fractal features are estimated from the decomposed time-frequency coefficients of IMFs. Estimated fractal features are arranged to represent different classes of bearing vibration activity. In order to estimate fractal information embedded in the time-frequency coefficients, Higuchi's algorithm of FDestimation is employed in present work.

HFD is an efficient algorithm for mesurement of fractal content of discrete time sequences and was proposed by Higuchi in $1988 .{ }^{49}$ For a given time series $S=s[1], s[2] \ldots \ldots, s[n]$, various steps involved in computation of HFD can be expressed as:

Form $k$ new time series $S_{k}^{m}$ from given time series as:

$$
\begin{aligned}
S_{k}^{m}= & \{s[m], s[m+k], s[m+2 k], \ldots \ldots \ldots, \\
& \left.s\left[m+i n t\left(\frac{N-m}{k}\right) \times k\right]\right\}
\end{aligned}
$$

where $k$ and $m$ are integers, $k$ indicates the discrete time interval between the points, and $m=1,2, \ldots, k$ represents initial time value. The length of each constructed time series is computed as (8) (see top of the previous page). ${ }^{51}$

Where $N$ is the length of the original time series $S$ and $\left[(N-1) /\left(\operatorname{int}\left(\frac{N-m}{k}\right) \times k\right)\right]$ is the normalization factor. The length of the curve for the time interval $k$ is defined as the average of $k$ values $L(m, k)$ for $m=1,2 \ldots, k$ and is given as:

$$
L(k)=\frac{1}{k} \times \sum_{m=1}^{k} L(m, k) ;
$$

when $L(k)$ is plotted against $1 / k$ on double logarithmic scale, with $k=1,2, \ldots, k_{\max }$, the data should fall on a straight line with a slope equal to the fractal dimension of HFD is defined as the slope of the line that fits the pairs $\{\ln [L(k)], \ln (1 / k)\}$ in least square sense. The value of $k_{\text {max }}$ is selected following the criteria suggested by Jindal et al. ${ }^{50}$ and Upadhyay et al. ${ }^{51}$

In order to select optimum IMFs for second level decomposition, a criterion of highest HFD values is introduced. Primarily, EMD is applied to the vibration signals for decomposition purpose. An EMD process decomposes vibration activity into set of IMFs and further, their HFD value is calculated. Initially, four IMFs are selected following highest HFD value criterion. Thereafter, a TQWT is applied on each selected IMFs. The TQWT decomposes each IMF into time-frequency sub-bands. Further, in order to prepare feature vector, HFD is estimated from each decomposed time-frequency sub-band of vibration activity and corresponding feature vector is prepared for each class. While calculating TQWT coefficients, the redundancy

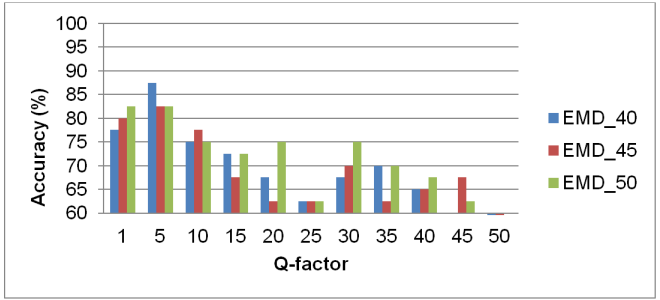

(a)

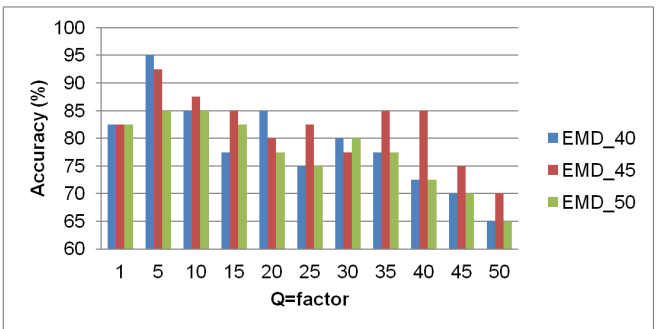

(b)

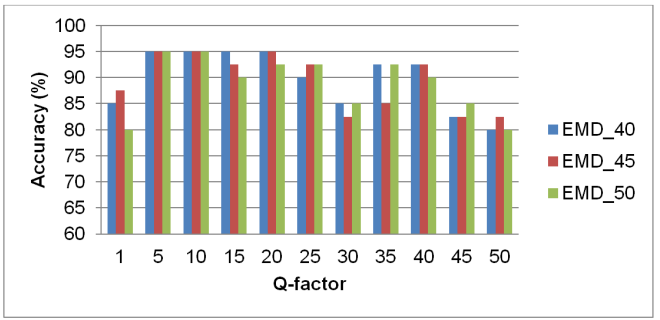

(c)

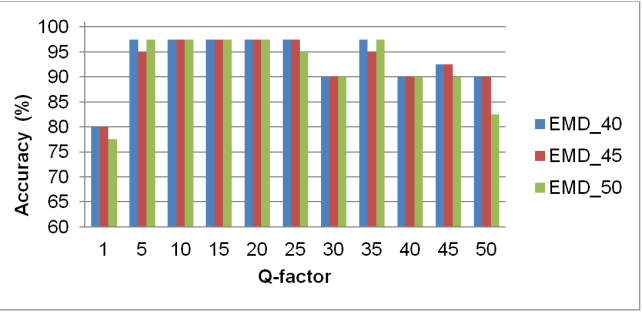

(d)

Figure 9. (a) SVM classification performance with varying values of parameters (feature vector size $=40$ features). (b) SVM classification performance with varying values of parameters (feature vector size $=30$ features). (c )SVM classification performance with varying values of parameters (feature vector size $=20$ features). (d) SVM classification performance with varying values of parameters (feature vector size $=10$ features).

parameter $r$ is taken as $r=3$ and number of decomposition levels are set to 9 which yields 10 sub-bands of decomposed vibration activity. The value of Q-factor is varied in the range of 1-50 and is optimized to attain the highest classification accuracy during fault diagnosis task. The obtained feature vectors are fed as an input to the soft computing technique for fault classification. Fault classification is performed using a SVM classifier on the WEKA toolkit. Different feature vectors are generated with different values of Q-factor and classification results are reported to attain highest classification efficiency. The proposed methodology of fault diagnosis is illustrated by schematic diagram presented in Fig 8.

\section{RESULTS AND DISCUSSION}

Feature vectors are prepared with varying parametric values and given as an input to a supervised machine learning technique, SVM, for training and validation purposes. Classification of vibrational activity is performed following 10-fold 


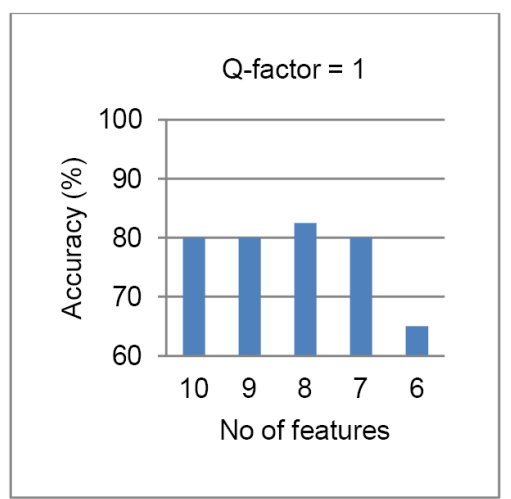

(a)

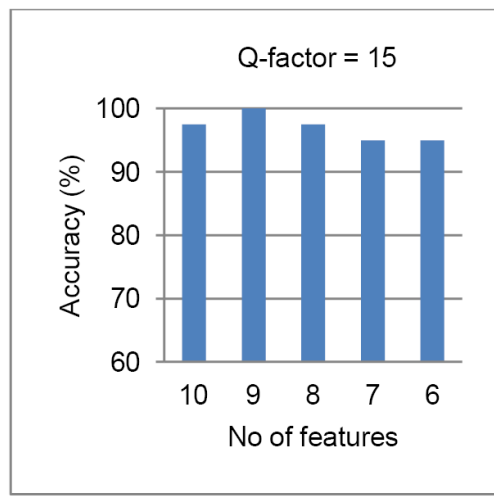

(d)

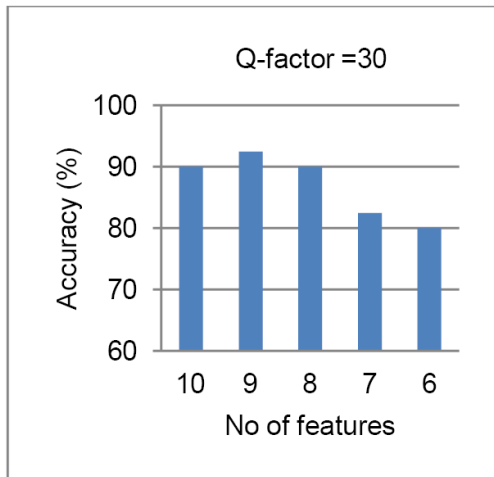

(g)

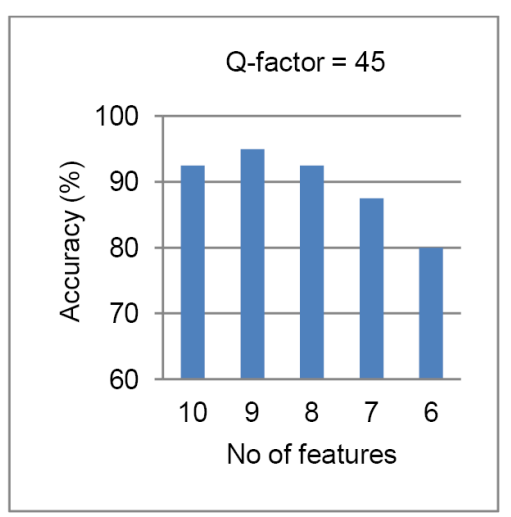

(j)

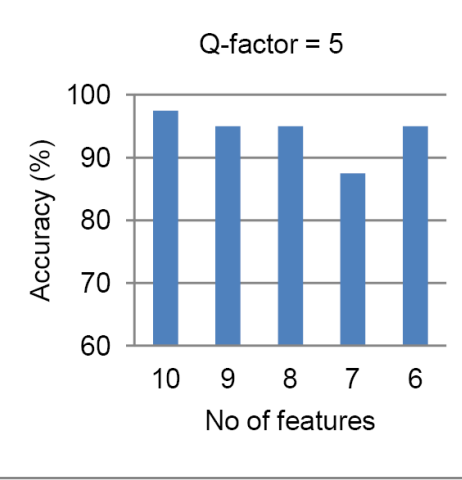

(b)

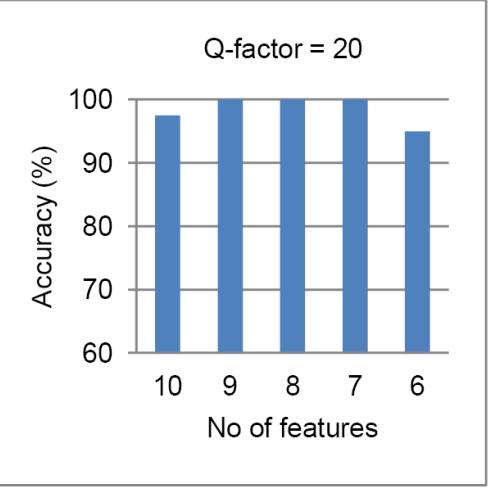

(e)

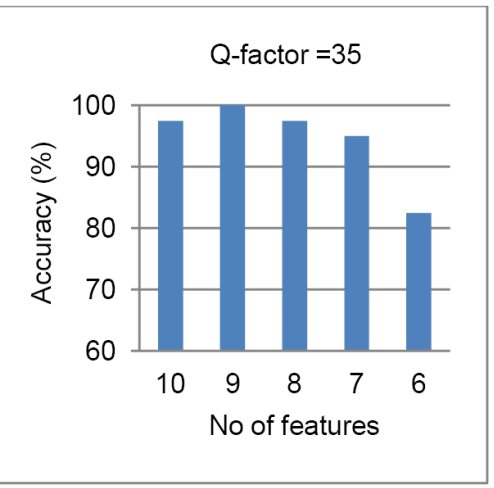

(h)

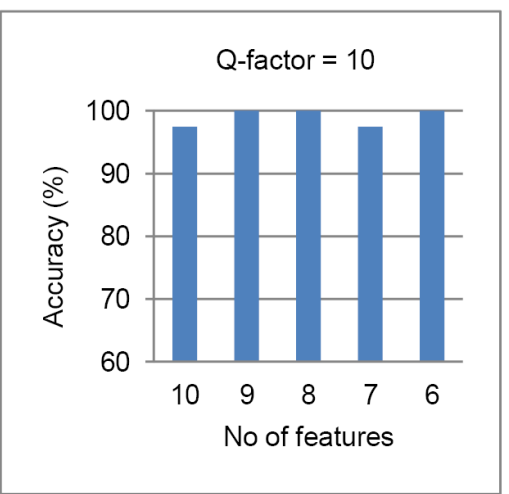

(c)

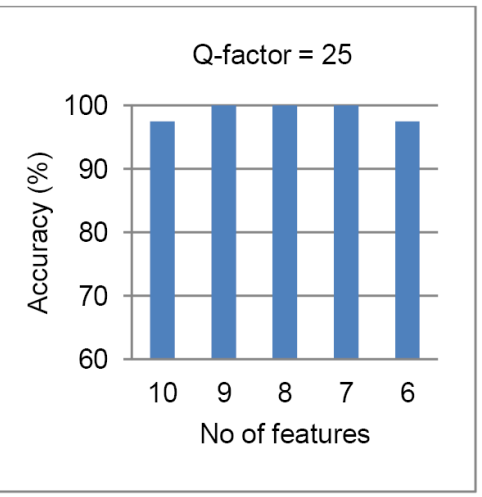

(f)

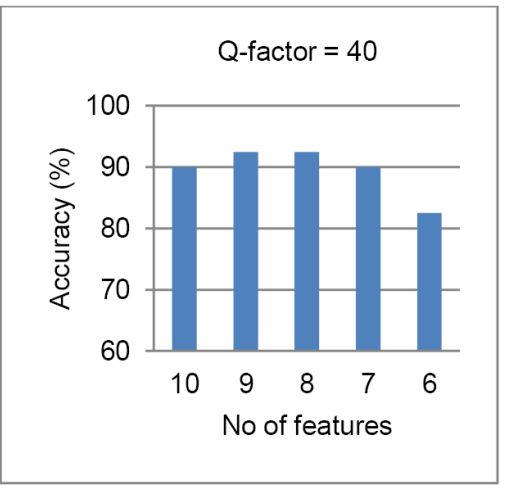

(i)

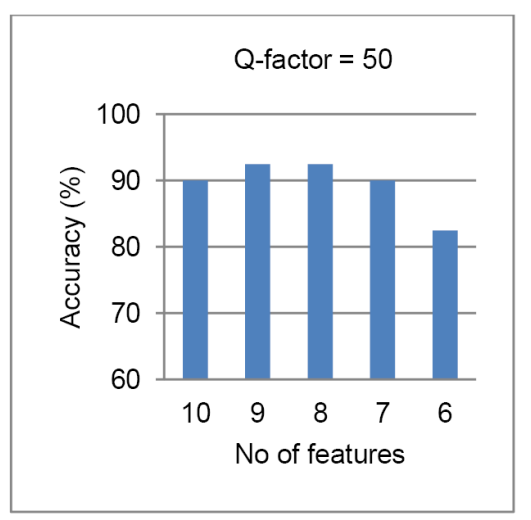

(k)

Figure 10. SVM classification performance with varying Q-factor and feature vector size (considering EMD_40 parameters) 


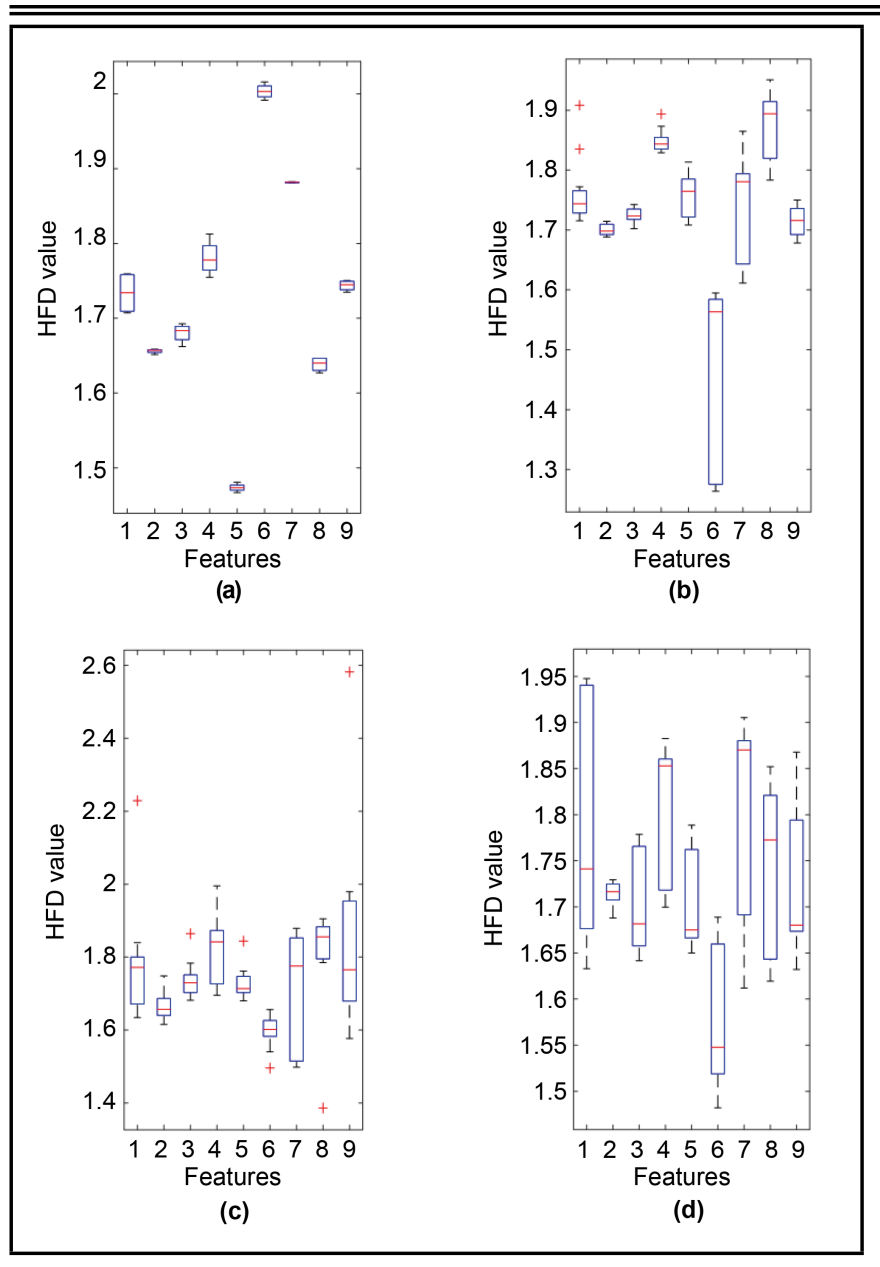

Figure 11. (a) Boxplot of EMD-TQWT based HFD features of healthy bearing. (b) Boxplot of EMD-TQWT based HFD features of faulty (ball defect) bearing. (c)Boxplot of EMD-TQWT based HFD features of faulty (outer race defect) bearing. (d)Boxplot of EMD-TQWT based HFD features of faulty (inner race defect) bearing.

cross validation approach to avoid statistical biasing. In 10fold cross validation approach, featured data is divided into ten folds and classification is performed in ten iterations. However, the final result is the average of all ten iterations. In the present work, parameters of EMD and TQWT are varied and classification results are analyzed against these parametric variations to attain the highest classification performance. To prepare feature vectors from recorded vibration activity, a total of 40 instances of raw vibration activity are considered, out of which 4 were of healthy bearing and 12 each were of IRD, ORD and BD. Following the criterion of highest HFD value, four feature vectors with varying vector sizes are prepared after calculating HFD features from four IMFs (40 features), three IMFs (30 features), two IMFs (20 features) and one IMF (10 features) respectively and ranking of the IMFs was carried out using HFD values. Training and validation of classifier algorithms is performed corresponding to all four feature vectors and classification performance is reported. Table 1 shows a sample input feature vector obtained after processing of a single IMF with the highest HFD value.

Figure 9(a-d) shows the classification performance of SVM classifier (i.e. classification accuracy) for different sets of feature vectors (feature vectors with 40 features, 30 features, 20 features and 10 features) with varying parametric values. Here, EMD_40, EMD_45 and EMD_50 imply that values of parameter qResol and qResid are 40, 45, and 50, respectively.
Table 2. Confusion matrix of 10-fold cross validation using SVM classifier $(Q=20$, number of features $=9)$.

\begin{tabular}{|c|c|c|c|c|}
\hline HB & BD & IRD & ORD & CLASSIFIED AS \\
\hline 4 & 0 & 0 & 0 & HB \\
\hline 0 & 12 & 0 & 0 & BD \\
\hline 0 & 0 & 12 & 0 & IRD \\
\hline 0 & 0 & 0 & 12 & ORD \\
\hline
\end{tabular}

HB: Healthy Bearing, BD: Ball Defect, IRD: Inner Race Defect, ORD: Outer Race Defect

While preparing classification results presented in the Fig. 9(a-d), the Q-factor values were varied in the range of 1-50 and step size was kept as 5. It is perceived from Fig. 9(ad) that classification accuracy first increases with the increase in Q-factor value and further decreases as Q-factor value approaches to 50. Classification accuracy seems constant over middle ranges of Q-factor values (i.e. 5 to 25) for feature vectors with smaller number of features, i.e., 20 features and 10 features (ref. Fig. 9(c) and Fig. 9(d)) and decreases afterwards. Also, it is evident from Fig. 9(a-d) that the classification performance improves with the reduction in number of HFD features and the highest classification accuracy is attained corresponding to least number of HFD features, i.e., 10. The EMD parameter variations have a smaller impact on the classifier's performance for a smaller feature set. However, keeping EMD parameter values as 40 helped in achieving a high classification rate in multiple experiments (ref. Fig. 9(d)).

The results of the classification established the fact that IMF with the highest value of HFD is the most significant and informative IMF for fault classification task. Considering efficacy of a single IMF with the highest value of HFD in a bearing fault diagnosis event, a second stage of feature reduction is carried out with different Q-factor values (ranging from 1-50) and the results are shown in Fig. 10(a-k). It is observed from Fig. 10(a-k) that reduction in the number of features significantly improved the classification performance, but further reduction yielded deteriorating impact on the classifier's performance. The best classification performance was recorded while considering features extracted from nine subbands of the decomposed IMF (ref. Fig. 10(c-k)). It is also observed that the classification accuracy is minimum for Qfactor $=1$ and it increases with increasing value of Q-factor until Q-factor is greater than 25. However, the best classification performance is witnessed when Q-factor value lies in the range of 10-25. Therefore, classification results suggest that the Q-factor value should be maintained in the range of 10-25 to ensure the highest classification performance in the bearing fault diagnosis task using the double decomposition technique.

Figure 11 $(a-d)$ represents the box plots of extracted HFD features obtained for four classes of vibration activity. It is observed from Fig. 11(a-d) that the distribution of feature values is much lower for a healthy bearing as compared to the bearings with faults. However, the maximum distribution of feature values is observed for bearing with ORD (ref. Fig. 11(d)). Figure 11(a-d) describes the class discrimination ability of the extracted EMD-TQWT based HFD features in bearing fault diagnosis using double decomposition technique. It is evident from Fig. 11(a-d) that the extracted HFD features have capability to differentiate among healthy and faulty vibration activity. Table 2 presents a confusion matrix for SVM classification; keeping Q-factor value as 20 and considering 9 features for training and validation purposes. It is reflected from Table 2 that all the instances are correctly classified using the SVM classifier. $q$ Resol and qResid are 40, 45, and 50, respectively. 
T. Dovedi, et al.: DIAGNOSIS OF BALL BEARING FAULTS USING DOUBLE DECOMPOSITION TECHNIQUE

Table 3. Classification performance achieved for drive end \& fan end vibration data (48 kHz sampling frequency).

\begin{tabular}{|c|c|c|c|c||c|c|c|c||}
\hline \multirow{2}{*}{ Parameter } & \multicolumn{7}{|c||}{ DATA (Sampling Frequency 48 kHz) } \\
\cline { 2 - 10 } Q-factor & \multicolumn{3}{|c|}{ DRIVE END } & \multicolumn{7}{c||}{ FAN END } \\
\hline Total number of instances & 40 & 40 & 40 & 40 & 40 & 40 & 40 & 40 \\
\hline Correctly classified instances & 40 & 40 & 40 & 40 & 36 & 34 & 34 & 36 \\
\hline Incorrectly classified instances & 0 & 0 & 0 & 0 & 4 & 6 & 6 & 4 \\
\hline Precision & 1 & 1 & 1 & 1 & 0.913 & 0.859 & 0.850 & 0.9 \\
\hline F- measure & 1 & 1 & 1 & 1 & 0.900 & 0.857 & 0.850 & 0.899 \\
\hline ROC Area & 1 & 1 & 1 & 1 & 0.904 & 0.887 & 0.873 & 0.898 \\
\hline Mean computational time & \multicolumn{3}{|c|}{$7.3 \mathrm{sec}$} & \multicolumn{7}{c}{$7.3 \mathrm{sec}$} & \\
\hline
\end{tabular}

Table 4. Classification performance achieved for drive end \& fan end vibration data ( $12 \mathrm{kHz}$ sampling frequency).

\begin{tabular}{|c|c|c|c|c|c|c|c|c|}
\hline \multirow[t]{2}{*}{ Parameter } & \multicolumn{8}{|c|}{ DATA (Sampling Frequency 12 kHz) } \\
\hline & \multicolumn{4}{|c|}{ DRIVE END } & \multicolumn{4}{|c|}{ FAN END } \\
\hline Q-factor & 10 & 15 & 20 & 25 & 10 & 15 & 20 & 25 \\
\hline Total number of instances & 40 & 40 & 40 & 40 & 39 & 39 & 39 & 39 \\
\hline Correctly classified instances & 40 & 39 & 37 & 35 & 37 & 35 & 35 & 36 \\
\hline Incorrectly classified instances & 0 & 1 & 3 & 5 & 2 & 4 & 4 & 3 \\
\hline Precision & 1 & 0.977 & 0.931 & 0.875 & 0.951 & 0.899 & 0.900 & 0.930 \\
\hline F-measure & 1 & 0.975 & 0.925 & 0.874 & 0.949 & 0.897 & 0.895 & 0.923 \\
\hline ROC Area & 1 & 0.984 & 0.944 & 0.934 & 0.963 & 0.945 & 0.937 & 0.927 \\
\hline Mean computational time & \multicolumn{4}{|c|}{$3.1 \mathrm{sec}$} & \multicolumn{4}{|c|}{$3.1 \mathrm{sec}$} \\
\hline
\end{tabular}

Further, in order to validate the efficacy of proposed feature extraction methodology, three vibration datasets with varying sampling frequency and sensor positioning (with sampling frequency $=12 \mathrm{kHz}$ at drive end \& fan end sensor positions; with sampling frequency $=48 \mathrm{kHz}$ at fan end sensor position) are exploit and a comparison of classification performance is carried out. Experiments are performed at four different Qfactor values (i.e., 10, 15, 20 and 25) and the number of features corresponding to each class were considered as 9. Since, EMD parameter variations have a smaller impact on classifier's performance for smaller feature set (as illustrated from Fig. 9(d)), EMD parameter values are kept 40 consistently across all experiments. Classification performance achieved for drive end \& fan end vibration data with a sampling frequency of $48 \mathrm{kHz}$ is presented in Table 3 . Classification performance achieved for drive end $\&$ fan end vibration data with a sampling frequency of $12 \mathrm{kHz}$ is presented in Table 4 . The classification accuracy obtained at varying Q-factor values (i.e. Q-factor=10/15/20/25) for four different vibration datasets is presented in Fig. 12. It is observed from Table 3 and Table 4 that the proposed methodology has the ability to identify faulty and healthy bearings using vibration activity recorded at varying sampling frequencies and sensor placement. The classification performance obtained for drive end vibration data is significantly better as compared to fan end vibration data. It is evident from Fig. 12 that best classification accuracy is obtained at Q-factor=10 for both $48 \mathrm{kHz}$ and $12 \mathrm{kHz}$ sampled vibration activity. A comparative study between the proposed work and previous work published in the literature is presented in Table 5. It is perceived from Table 5 that the methodology proposed in the present work performed better than previously proposed work in terms of classification performance. Double decomposition based HFD feature estimation method helped in achieving $100 \%$ classification accuracy with a significantly lower number of features.

\section{CONCLUSION}

In present work, an effort has been made to develop EMDTQWT based double decomposition technique for the fault diagnosis of ball bearing. The proposed technique helps in identifying ball defect, inner race defect and outer race defect present in ball bearing. The methodology relies on EMDTQWT based time-frequency representation of bearing vibration activity for calculation of HFD features as discriminable signatures of faults. It is observed in the study that HFD boxplots have significant distribution over values corresponding to vibration activity of faulty bearings, however, such is not the case with healthy bearings. The box-plot of HFD features for healthy bearing shows positive/zero skewness pattern, however, mixed skewness pattern is witnessed for faulty bearings. The four classes of vibration activity is classified using SVM classifier with varying size of feature vector and parametric values. The results of classification illustrated the significance of IMF with the highest value of HFD in fault diagnosis task. Also, very substantial variation in classification accuracy is discerned with varying value of Q-factor in this work. It is recommended that Q-factor value should be maintained in the range of 10-25 to ensure high classification performance. The highest classification accuracy of $100 \%$ is achieved corresponding to Q-factor values of 10, 15, 20, and 25 with 9 HFD features in the present work (for $48 \mathrm{kHz}$, drive end data). It is observed that increment and decrement in the number of features had negative impact on the classification performance. The results of classification illustrates the efficacy of the proposed double decomposition technique in bearing fault diagnosis. The effectiveness of the proposed methodology is further evaluated on three different vibration datasets and classification performance is analyzed. Considering all other parameters as constant, the highest classification accuracy of $100 \%$ is achieved for $12 \mathrm{kHz}$, drive end vibration data. Comparison results with previous studies revealed that proposed feature extraction methodology has capabilities to identify bearing faults with a significantly lower number of estimated features.

\section{REFERENCES}

1 Kankar, P.K., Sharma, S.C., and Harsha, S.P. Fault diagnosis of ball bearings using machine learning methods, $E x$ pert Systems with applications, 38(3), 1876-886, (2011). https://dx.doi.org/10.1016/j.eswa.2010.07.119

2 Martin, H.R. and Honarvar, F. Application of statisti- 
Table 5. A comparative study between proposed work and previous work published in the literature.

\begin{tabular}{||c|c|c|c|c||}
\hline Authors & Number of featureused & Classifiers used & Peak efficiency & Remark \\
\hline Wu et al. & Eighty (statistical) & SVM & $98.5 \%$ & Training efficiency \\
\hline Zhang and Li $^{53}$ & Twenty & $\begin{array}{c}\text { Neighborhood preserving } \\
\text { embedding and SOM }\end{array}$ & $99 \%$ & Training efficiency \\
\hline Tiwari et al. $^{54}$ & sixteen & ANFC & 92.50 & Ten-fold cross-validation efficiency \\
\hline Vakharia et al. $^{55}$ & Eleven & ANN, SVM & ANN - 97.5 SVM - 97.5 & Ten-fold cross-validationefficiency \\
\hline Wang et al. $^{56}$ & Hundred & CART, kNN and SVM & $97.26 \%$ & - \\
\hline Li et al. & One hundred eighty & SVM-BT & $100 \%$ & Training efficiency \\
\hline Vakharia et al. & Thirty-five (statistical) & SVM and RF & $98.38 \%$ (using RF) & Ten-fold cross-validation efficiency \\
\hline Present work & Nine & SVM & $100 \%$ (Q-factor=10) & Ten-fold cross-validation efficiency \\
\hline
\end{tabular}

SVM: Support Vector Machine, CART: Classification and Regression Trees, kNN: k-Nearest Neighbour, ANN: Artificial Neural Network, RF: Random Forest, ANFC: Adaptive Neuro Fuzzy Classifier

cal moments to bearing failure detection, Applied Acoustics, 44(1), 67-77, (1995). https://dx.doi.org/10.1016/0003$682 \times(94) \mathrm{p} 4420-\mathrm{b}$

3 Honarvar, F. and Martin, H.R. New statistical moments for diagnostics of rolling element bearings, Journal of Manufacturing Science Engineering, 119(3), 425-32, (1997). https://dx.doi.org/10.1115/1.2831123

4 Mechefske, C.K. and Mathew, J. Fault detection and diagnosis in low speed rolling element bearings part I: the use of parametric spectra, Mechanical Systems and Signal Processing, 6(4), 297-307, (1992). https://dx.doi.org/10.1016/0888-3270(92)90032-e

$5 \mathrm{Li}, \mathrm{L}$. and Qu, L. Cyclic statistics in rolling bearing diagnosis, Journal of Sound Vibration, 267(2), 253-65, (2003). https://dx.doi.org/10.1016/s0022-460x(02)01412-8

6 Zvokelj, M., Zupan, S. and Prebil, I. Multivariate and multiscale monitoring of large-size low-speed bearings using ensemble empirical mode decomposition method combined with principal component analysis, Mech Syst Signal Process, 24(4), 1049-67, (2010). https://dx.doi.org/10.1016/j.ymssp.2009.09.002

7 Mori, K., Kasashima, N., Yoshioka, T., and Ueno, Y. Prediction of spalling on a ball bearing by applying the discrete wavelet transform to vibration signals, Wear, 195(1), 162-168, (1996). https://dx.doi.org/10.1016/00431648(95)06817-1

8 Seker, S. and Ayaz, E. Feature extraction related to bearing damage in electric motors by wavelet analysis, Journal of Frankl Inst, 340(2), 125-134, (2003). https://dx.doi.org/10.1016/s0016-0032(03)00015-2

9 Lou, X. and Loparo, K.A. Bearing fault diagnosis based on wavelet transform and fuzzy inference, Mechanical Systems Signal Process, 18(5), 1077-1095, (2004). https://dx.doi.org/10.1016/s0888-3270(03)00077-3

10 Yang, Y., Yu, D. and Cheng, J. A fault diagnosis approach for roller bearing based on IMF envelope spectrum and SVM, Measurement, 40(9), 943-950, (2007). https://dx.doi.org/10.1016/j.measurement.2006.10.010

11 He, W., Jiang, Z.N., and Feng, K. Bearing fault detection based on optimal wavelet filter and sparse code shrinkage, Measurement, 42(7), 1092-1102, (2009). https://dx.doi.org/doi.org/10.1016/j.measurement.2009.04.001

12 Van, W.B.J., Van, W.M.A., and Qi, G. Difference histograms: a new tool for time series analysis applied to bearing fault diagnosis, Pattern Recognition Letters, 30(6), 595-599, (2009). https://dx.doi.org/10.1016/j.patrec.2008.12.012
13 Hong, H. and Liang, M. Fault severity assessment for rolling element bearings using the LempelZiv complexity and continuous wavelet transform, Journal of Sound Vib, 320(1), 452-468, (2009). https://dx.doi.org/10.1016/j.jsv.2008.07.011

14 Zhao, S., Liang, L., Xu, G., Wang, J., and Zhang, W. Quantitative diagnosis of a spall-like fault of a rolling element bearing by empirical mode decomposition and the approximate entropy method, Mechanical System Signal Process, 40(1), 154-177, (2013). https://dx.doi.org/10.1016/j.ymssp.2013.04.006

15 Jacek, D. and Radosław, Z. Rolling bearing diagnosing method based on Empirical Mode Decomposition of machine vibration signal, Applied Acoustics, 77, 195-203, (2014). https://dx.doi.org/10.1016/j.apacoust.2013.09.001

16 Lium H. and Han, M. A fault diagnosis method based on local mean decomposition and multi-scale entropy for roller bearings, Mech Mach Theory, 75, 67- 78, (2014). https://dx.doi.org/10.1016/j.mechmachtheory.2014.01.011

17 Patel, V.N., Tandon, N., and Pandey, R.K. Defect detection in deep groove ball bearing in presence of external vibration using envelope analysis and Duffing oscillator, Measurement, 45(5), 960-970, (2012). https://dx.doi.org/10.1016/j.measurement.2012.01.047

18 Klein, R., Masad, E., Rudyk, E., and Winkler, I. Bearing diagnostics using image processing methods, Mech Syst Signal Process, 45(1), 105-113, (2014). https://dx.doi.org/10.1016/j.ymssp.2013.10.009

19 Ming, A.B., Qin, Z.Y., Zhang, W., and Chu, F.L. Spectrum auto-correlation analysis and its application to fault diagnosis of rolling element bearings, Mechanical System Signal Process, 41(1), 141-154, (2013). https://dx.doi.org/10.1016/j.ymssp.2013.08.004

20 Lei, Y., Lin, J., He, Z., and Zi, Y. Application of an improved kurtogram method for fault diagnosis of rolling element bearings, Mechanical System Signal Process, 25(5), 1738-1749, (2011). https://dx.doi.org/10.1016/j.ymssp.2010.12.011

$21 \mathrm{Li}, \mathrm{H}$., Xu, F., Liu, H., and Zhang, X. Incipient fault information determination for rolling element bearing based on synchronous averaging reassigned wavelet scalogram, Measurement, 65, 1-10, (2015). https://dx.doi.org/10.1016/j.measurement.2014.12.032

22 Yang, J., Zhang, Y., and Zhu, Y. Intelligent fault diagnosis of rolling element bearing based on SVMs and fractal dimension, Mech. Syst. Signal Process, 21(5), 2012-2024, (2007). https://dx.doi.org/10.1016/j.ymssp.2006.10.005 


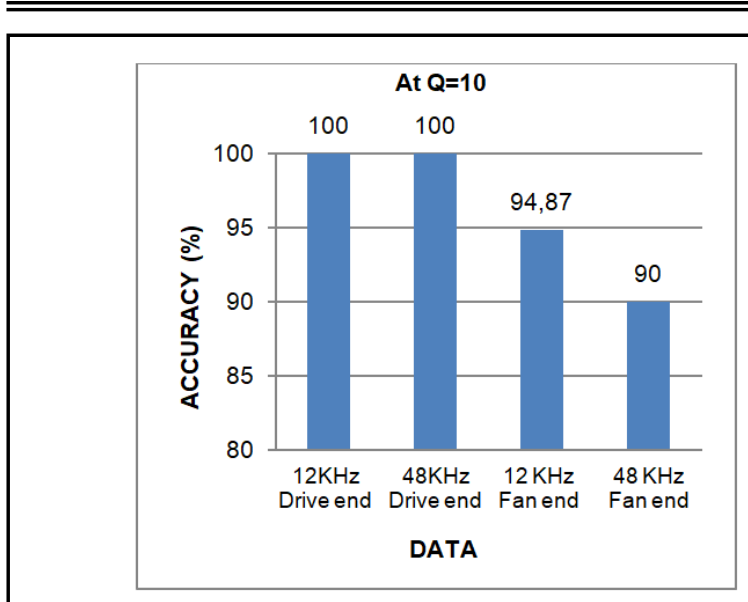

(a)

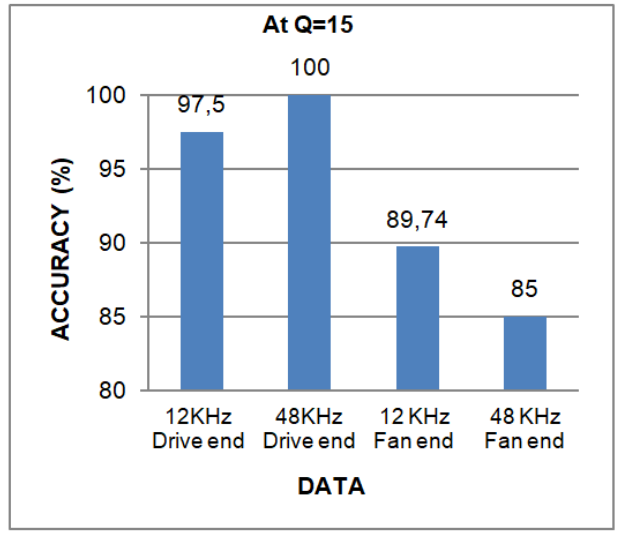

(b)

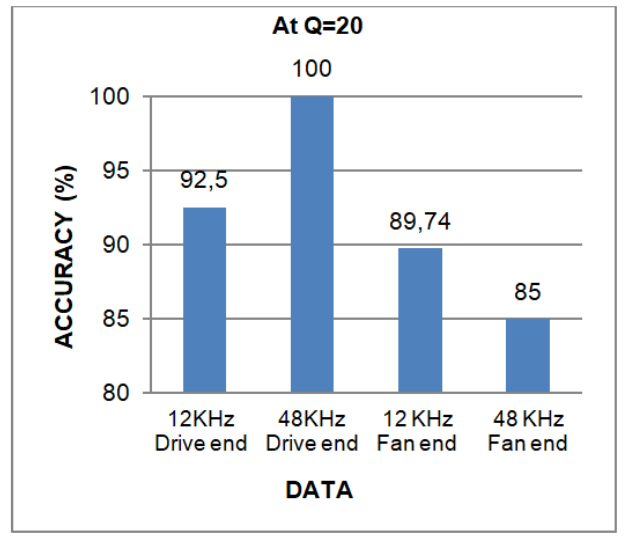

(c)

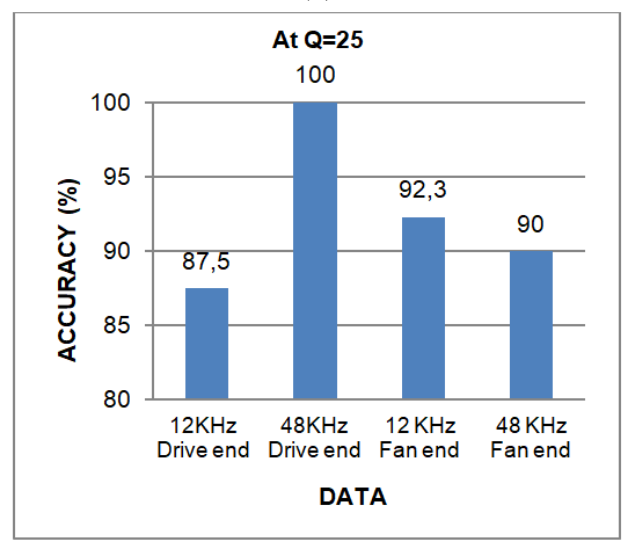

(d)

Figure 12. Classification accuracy obtained at $Q=10 / 15 / 20 / 25$ for four different vibration dataset.
23 Yan, R. and Gao, R.X. Approximate Entropy as a diagnostic tool for machine health monitoring, Mechanical Systems and Signal Processing, 21(2), 824-839, (2007). https://dx.doi.org/10.1016/j.ymssp.2006.02.009

24 Zheng, J., Cheng, J., and Yang, Y. Multiscale permutation entropy based rolling bearing fault diagnosis, Shock Vib., 1-8, (2014). https://dx.doi.org/10.1155/2014/154291

25 Zhang, L., Xiong, G., Liu, H. et al. Bearing fault diagnosis using multiscale entropy and adaptive neuro-fuzzy inference, Expert Syst. Appl., 37(8), 6077-6085, (2010). https://dx.doi.org/10.1016/j.eswa.2010.02.118

26 Liang, M. and Faghidi, H. Intelligent bearing fault detection by enhanced energy operator, Expert Systems with Applications, 41, 7223-7234, (2014). https://dx.doi.org/10.1016/j.eswa.2014.05.026

27 Caesarendra, W., Kosasih, B., Tieu, A. K., et al. Application of the largest Lyapunov exponent algorithm for feature extraction in low speed slew bearing condition monitoring, Mechanical Systems and Signal Processing, 50-51, 116-138 (2015). https://dx.doi.org/10.1016/j.ymssp.2014.05.021

28 Boškoski, P., Gašperin, M., Petelin, D., et.al. Bearing fault prognostics using Rényi entropy based features and Gaussian process models, Mechanical Systems and Signal Processing, 52-53, 327-337 (2015). https://dx.doi.org/10.1016/j.ymssp.2014.07.011

29 Liu, H., Wang, X., and Lu, C. Rolling bearing fault diagnosis based on LCD-TEO and multifractal detrended fluctuation analysis, Mechanical Systems and Signal Processing, 60-61, 273-288 (2015). https://dx.doi.org/10.1016/j.ymssp.2015.02.002

30 Han, L., Li, C.W., Guo, S. L. et.al. Feature extraction method of bearing AE signal based on improved FAST-ICA and wavelet packet energy, Mechanical Systems and Signal Processing, 62-63, 91-99 (2015). https://dx.doi.org/10.1016/j.ymssp.2015.03.009

31 He, D., Wang, X., Li, S. et.al. Identification of multiple faults in rotating machinery based on minimum entropy deconvolution combined with spectral kurtosis, Mechanical Systems and Signal Processing, 81, 235-249 (2016). https://dx.doi.org/10.1016/j.ymssp.2016.03.016

32 Zhang, H., Chen, X., Du, Z., et.al. Kurtosis based weighted sparse model with convex optimization technique for bearing fault diagnosis, Mechanical Systems and Signal Processing, 80, 349-376 (2016). https://dx.doi.org/10.1016/j.ymssp.2016.04.033

33 Geoff, L. McDonald, and Zhao, Q. Multipoint Optimal Minimum Entropy Deconvolution and Convolution Fix: Application to vibration fault detection, Mechanical Systems and Signal Processing, 82, 461-477 (2017). https://dx.doi.org/10.1016/j.ymssp.2016.05.036

34 Zheng, J., Pan, H., and Cheng, J. Rolling bearing fault detection and diagnosis based on composite multiscale fuzzy entropy and ensemble support vector machines, Mechanical Systems and Signal Processing, 85, 746-759, (2017). https://dx.doi.org/10.1016/j.ymssp.2016.09.010 
35 Ai, Y., Guan, J., Fei, C., et.al. Fusion information entropy method of rolling bearing fault diagnosis based on n-dimensional characteristic parameter distance, Mechanical Systems and Signal Processing, 88, 123-136 (2017). https://dx.doi.org/10.1016/j.ymssp.2016.11.019

36 Miao, Y., Zhao, M., and Lin, J. Application of an improved maximum correlated kurtosis deconvolution method for fault diagnosis of rolling element bearings, Mechanical Systems and Signal Processing, 92, 173-195, (2017). https://dx.doi.org/10.1016/j.ymssp.2017.01.033

37 Selesnick, I.W. Wavelet Transform with Tunable Q-factor, IEEE Transactions on Signal Processing, 59, 3560-3575, (2011). https://dx.doi.org/10.1109/tsp.2011.2143711

38 Gaigai, C., Xuefeng, C., and Zhengjia, H. Sparsity-enabled signal decomposition using Tunable Q-factor Wavelet Transform for fault feature extraction of gearbox, Mechanical Systems and Signal Processing, 41, 34-53, (2013). https://dx.doi.org/10.1016/j.ymssp.2013.06.035

39 He, W., Zi, Y., Chen, B., Wu, F., and He, Z. Automatic fault feature extraction of mechanical anomaly in induction motor bearing using ensemble super-wavelet transform, Mech. Syst. And Signal Process, 54-55, 457-480, (2015). https://dx.doi.org/10.1016/j.ymssp.2014.09.007

40 Kumar, A., Prakash, A., and Kumar, R. Tunable Q-factor wavelet transform for extraction of weak bursts in the vibration signal of an angular contact bearing, Procedia Tech., 25, 838-845, (2016). https://dx.doi.org/10.1016/j.protcy.2016.08.188

41 Huang, N.E., Shen, Z., Long, S.R., Wu, M.C., Shih, H.H., and Zheng, Q. The Empirical Mode Decomposition and the Hilbert spectrum for nonlinear and non-stationary time series analysis, Proc Royal Soc London, 454, 903-95, (1998). https://dx.doi.org/10.1098/rspa.1998.0193

42 Kumari, S., Upadhyay, R., and Padhy, P.K., Kankar P.K. Application of empirical mode decomposition for feature extraction from EEG signals, 2015 International Conference on Computer, Communication and Control (IC4), 1-6, (2013). https://dx.doi.org/ieeexplore.ieee.org/abstract/document/7375

43 Dybala, J., and Zimroz, R. Rolling bearing diagnosing method based on empirical mode decomposition of machine vibration signal, Applied Acoustics, 77, 195-203, (2014). https://dx.doi.org/10.1016/j.apacoust.2013.09.001

44 Dong, G., Chen, J. Noise resistant time frequency analysis and application in fault diagnosis of rolling element bearings, Mechanical System Signal Process, 33, 212-236, (2012). https://dx.doi.org/10.1016/j.ymssp.2012.06.008

45 Lei, Y., Lin, J., He, Z., and Zuo, M.J. A review on empirical mode decomposition in fault diagnosis of rotating machinery, Mechanical System Signal Process, 35, 108-26, (2013). https://dx.doi.org/10.1016/j.ymssp.2012.09.015

46 Rato, R.T., Ortigueira, M.D., and Batista, A.G. On the HHT, its problems, and some solutions, Mechanical Systems and Signal Processing, 22(6), 1374-1394, (2008). https://dx.doi.org/10.1016/j.ymssp.2007.11.028
47 Mercy, M.S. Performance Analysis of Epileptic Seizure Detection Using DWT \& ICA with Neural Networks. International Journal of Analog Integrated Circuits and Signal Processing, 2(4), 1109-1113, (2014).

48 Jindal, K., Upadhyay, R., and Singh, H.S. Application of tunable-Q wavelet transform based nonlinear features in epileptic seizure detection, Analog Integrated Circuits and Signal Processing, 100(2), 437-452, (2019). https://dx.doi.org/10.1007/s10470-019-01424-y

49 Higuchi, T. Approach to an irregular time series on the basis of the fractal theory, Physica D, 31, 277-283, (1988). https://dx.doi.org/10.1016/0167-2789(88)90081-4

50 Jindal, K., Upadhyay, R., Singh, H.S., Vijay, M., Sharma, A., Gupta, K., Gupta, J., and Dube, A. Migraine disease diagnosis from EEG signals using Non-linear Feature Extraction Technique, 2018 IEEE International Conference on Computational Intelligence and Computing Research (ICCIC), 1-4, (2018). https://dx.doi.org/10.1109/ICCIC.2018.8782341

51 Upadhyay, R., Manglick, A., Reddy, D.R., Padhy, P.K., and Kankar, P.K. Channel optimization and nonlinear feature extraction for Electroencephalogram signals classification, Computers and Electrical Engineering, 45, 222-234, (2015). https://dx.doi.org/10.1016/j.compeleceng.2015.03.015

$52 \mathrm{Wu}$, S.D., Wu, C.W., Wu, T.Y., and Wang, C.C. Multi-scale analysis based ball bearing defect diagnostics using Mahalanobis distance and support vector machine, Entropy, 15(2), 416-433, (2013). https://dx.doi.org/10.3390/e15020416

53 Zhang, S. and Li, W. Bearing condition recognition and degradation assessment under varying running conditions using NPE and SOM, Math Probl Eng., 2014, 1-10, (2014). https://dx.doi.org/10.1155/2014/781583

54 Tiwari, R., Gupta, V.K., and Kankar, P.K. Bearing fault diagnosis based on multiscale permutation entropy and adaptive neuro fuzzy classifier, Journal of vibration and control, 21(3), 461-467, (2015). https://dx.doi.org/10.1177/1077546313490778

55 Vakharia, V., Gupta, V.K., and Kankar, P.K. A mul08 tiscale permutation entropy based approach to select wavelet for fault diagnosis of ball bearings, Journal of Vibration and Control, 21(16), 3123-3131, (2015). https://dx.doi.org/10.1177/1077546314520830

56 Wang, X., Zheng, Y., Zhao, Z., and Wang, J. Bearing fault diagnosis based on statistical locally linear embedding, Sensors 15(7), 16225-16247, (2015). https://dx.doi.org/10.3390/s150716225

57 Li, Y., Xu, M., Wei, Y., and Huang, W. A new rolling bearing fault diagnosis method based on multiscale permutation entropy and improved support vector machine based binary tree, Measurement, 77, 80-94, (2016). https://dx.doi.org/10.1016/j.measurement.2015.08.034

58 Vakharia, V., Gupta, V.K., and Kankar, P.K. Efficient fault diagnosis of ball bearing using ReliefF and Random Forest classifier, Journal of the Brazilian Society of Mechanical Sciences and Engineering, 39(8), 2969-2982, (2017). https://dx.doi.org/10.1007/s40430-017-0717-9 